\title{
Remimazolam: pharmacological characteristics and clinical applications in anesthesiology
}

Received December 3, 2021

Revised December 27, 2021

Accepted December 29, 2021

\author{
Corresponding author \\ Kyung Mi Kim, M.D., Ph.D. \\ Department of Anesthesiology and \\ Pain Medicine, Asan Medical Center, \\ University of Ulsan College of \\ Medicine, 88 Olympic-ro 43-gil, \\ Songpa-gu, Seoul 05505, Korea \\ Tel: 82-2-3010-5631 \\ Fax: 82-2-3010-6790 \\ E-mail: sumsonyo@gmail.com
}

\section{Kyung Mi Kim}

Department of Anesthesiology and Pain Medicine, Asan Medical Center, University of Ulsan College of Medicine, Seoul, Korea
A novel ultra-short-acting benzodiazepine (BDZ), remimazolam (CNS 7056), has been designed by 'soft drug' development to achieve a better sedative profile than that of the current drugs. Notably, the esterase linkage in remimazolam permits rapid hydrolysis to inactivate metabolites by non-specific tissue esterase and induces a unique and favorable pharmacological profile, including rapid onset and offset of sedation and a predictable duration of action. Similar to other BDZs, its sedative effects can be reversed using flumazenil, a BDZ antagonist. The pharmacokinetics and pharmacodynamics of remimazolam are characterized by relatively high clearance, small steady-state volume of distribution, short elimination half-life, short context-sensitive half-life, and fast onset and recovery, indicating rapid elimination, minimal tissue accumulation, and good control. In addition, remimazolam possesses a superior safety profile, including low liability for cardiorespiratory depression and injection pain, making it a preferred hypnotic agent in various clinical settings. Early clinical investigations suggest that remimazolam is well tolerated and effective for procedural sedation and for induction and maintenance of general anesthesia. To date, however, the clinical use of remimazolam has been confined to a few volunteer studies and a limited number of clinical investigations. Therefore, further studies regarding its recovery issues or postoperative complications, characteristics of electroencephalogram changes, and cost-benefit analyses are required to facilitate its widespread use.

Keywords: Benzodiazepine; Hypnotic; Pharmacodynamics anesthesia; Pharmacokinetics; Remimazolam; Sedation.

\section{INTRODUCTION}

Remimazolam besylate (Byfavo injection in South Korea, Anerem ${ }^{\circledR}$ in Japan, Aptimyda ${ }^{\mathrm{TM}}$ in EU, ByFavo ${ }^{\mathrm{TM}}$ in the USA, and Ruima ${ }^{\circledR}$ in China) is a water-soluble, ultra-short-acting intravenous (IV) benzodiazepine (BDZ). It has recently been approved as a general anesthetic (in January 2020 in Japan and January 2021 in South Korea) and for use in procedural sedation (in July 2020 in the USA and China, March 2021 in Europe, and August 2021 in South Korea) in adults [1].
Similar to midazolam, remimazolam enhances $\gamma$-aminobutyric acid $\mathrm{A}\left(\mathrm{GABA}_{\mathrm{A}}\right)$ receptor activity to induce cell membrane hyperpolarization, thereby inhibiting neural activity via an increase in chloride influx [2]. In addition, it is a soft drug designed to incorporate a carboxylic ester moiety into the BDZ core (Fig. 1) [3]. With such structural modifications similar to remifentanil, remimazolam is rapidly hydrolyzed to a pharmacologically inactive metabolite (CNS 7054) via non-specific tissue esterase activity (Fig. 2) [4,5], which leads to the fast onset and offset of sedation and predictable dura-

This is an Open Access article distributed under the terms of the Creative Commons Attribution Non-Commercial License (http://creativecommons.org/licenses/by-nc/4.0) which permits unrestricted non-commercial use, distribution, and reproduction in any medium, provided the original work is properly cited.

Copyright (C) the Korean Society of Anesthesiologists, 2022 
tion of action. Similar to other BDZs, flumazenil can be used to reverse the sedation effect of remimazolam [6]. Owing to its favorable properties, including rapid onset, organ-independent metabolism, short duration of action, predictable recovery, availability of a reversal agent, and a superior safety profile similar to other BDZs in terms of hemodynamic stability, remimazolam appears to have several advantages over currently available short-acting sedative drugs $[1,7]$.

In this review, the pharmacokinetic and pharmacodynamic characteristics and clinical applications of remimazolam have been discussed along with the currently available literary evidence.

\section{PHARMACOLOGICAL CHARACTERISTICS}

\section{Pharmacokinetics}

The pharmacokinetic properties of IV remimazolam have been investigated in previous phase I studies involving healthy volunteers administered single bolus injection $[8,9]$ and continuous infusion $[10,11]$, and it was found that remimazolam exhibits a relatively high clearance, a small steadystate volume of distribution $\left(\mathrm{V}_{\mathrm{ss}}\right)$, a short elimination half-

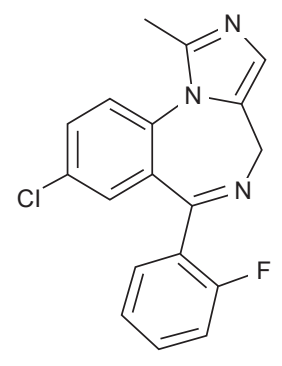

Midazolam

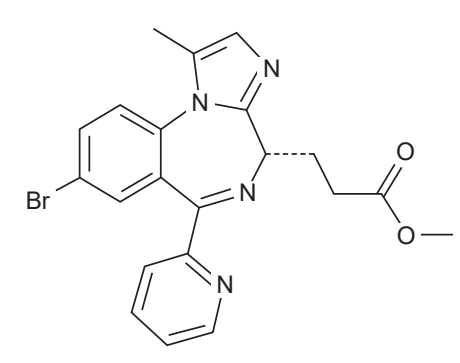

Remimazolam
Fig. 1. Molecular structures of midazolam and remimazolam [3]. life, a short context-sensitive half-life (CSHT), first-order, and linear pharmacokinetics. The major pharmacokinetic properties of remimazolam are summarized in Table 1.

The initial phase I dose-finding study, the first human trial of remimazolam, was conducted to compare the pharmacokinetics of remimazolam (0.01-0.30 $\mathrm{mg} / \mathrm{kg}$ administered over $1 \mathrm{~min})$ with that of midazolam $(0.075 \mathrm{mg} / \mathrm{kg}$ administered over $1 \mathrm{~min}$ ) [8]. The mean $\mathrm{V}_{\mathrm{ss}}$ of remimazolam was 34.8 $\mathrm{L}$, whereas that of midazolam was $81.8 \mathrm{~L}$. In addition, the elimination clearance of remimazolam was approximately three times that of midazolam (70.3 vs. $23.0 \mathrm{~L} / \mathrm{h})$ and independent of body weight. The mean residence times of remimazolam and midazolam were $0.51 \mathrm{~h}$ and $3.62 \mathrm{~h}$, respectively; the terminal half-life values were $0.75 \mathrm{~h}$ and $4.29 \mathrm{~h}$, respectively. A subsequent pharmacokinetic analysis that included data from 20 healthy male volunteers receiving continuous remimazolam infusion $(5 \mathrm{mg} / \mathrm{min}$ for $5 \mathrm{~min}$, followed by $3 \mathrm{mg} / \mathrm{min}$ for $15 \mathrm{~min}$ and $1 \mathrm{mg} / \mathrm{min}$ for $15 \mathrm{~min}$ ) showed similar profiles to those of an earlier phase I study involving IV bolus injection of remimazolam [10]. The analysis revealed a small $\mathrm{V}_{\mathrm{ss}}(35.4 \mathrm{~L} / \mathrm{h})$, high elimination clearance (1.15 L/min), short half-life (70 $\mathrm{min})$, and first-order linear pharmacokinetics.

Remimazolam is rapidly and extensively metabolized by tissue esterase (chiefly, liver carboxylesterase) to a pharmacologically inactive carboxy acid metabolite (CNS 7054), which has approximately 300 times lower affinity than that of its parent compound $[5,12,13]$. Simultaneous pharmacokinetic analysis of CNS 7054 and remimazolam revealed that CNS 7054 has a pharmacokinetic profile with smaller volume of distribution, slower clearance rate, and longer mean residence time than those of remimazolam [10]. In addition, a recent trial using a $3 \mathrm{D}$ bioreactor system to investigate the long-term stability of remimazolam metabolism in human liver cells demonstrated that continuous infusion of remimazolam over 5 days exhibited a stable metabolism and had

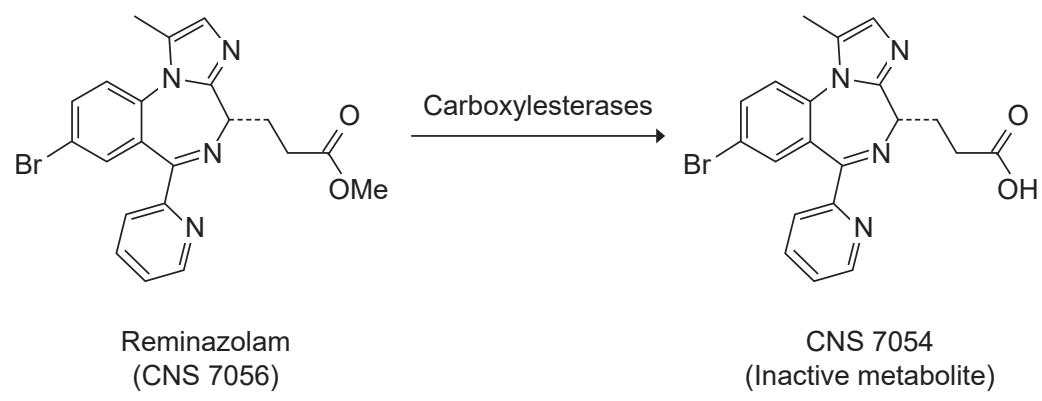

Fig. 2. Metabolism of remimazolam (CNS 7056) to the inactive metabolite (CNS 7054) [5]. 
Table 1. Summary of the Major Pharmacokinetic Parameters of Remimazolam in Healthy Volunteers

\begin{tabular}{|c|c|c|c|c|c|c|c|}
\hline Study (yr) & $\begin{array}{c}\text { Study } \\
\text { population }\end{array}$ & $\begin{array}{l}\text { Treatment } \\
\text { group }\end{array}$ & $\begin{array}{c}\text { Remimazolam dose and } \\
\text { duration }\end{array}$ & PK model & $\mathrm{V}_{\mathrm{ss}}(\mathrm{L})$ & $\mathrm{CL}(\mathrm{L} / \mathrm{min})$ & $\begin{array}{l}\text { Terminal elimi- } \\
\text { nation half-time } \\
\text { (min) }\end{array}$ \\
\hline $\begin{array}{l}\text { Antonik et al. } \\
(2012)[8] \\
\text { Wiltshire et al. } \\
(2012)[17]\end{array}$ & $\begin{array}{l}\text { Healthy } \\
\text { volunteers } \\
(n=81)\end{array}$ & $\begin{array}{l}\text { Remimazolam } \\
\text { Midazolam } \\
\text { Placebo }\end{array}$ & $\begin{array}{l}0.01-0.3 \mathrm{mg} / \mathrm{kg} \\
1 \mathrm{~min}\end{array}$ & $\begin{array}{l}\text { Recirculatory } \\
\text { model }\end{array}$ & $34.8 \pm 9.4$ & $1.17 \pm 0.23$ & $45.0 \pm 9.0$ \\
\hline $\begin{array}{l}\text { Zhou et al. } \\
\qquad(2018)[9]^{*}\end{array}$ & $\begin{array}{l}\text { Healthy } \\
\text { volunteers } \\
(n=79)\end{array}$ & $\begin{array}{l}\text { Remimazolam } \\
\text { Midazolam }\end{array}$ & $\begin{array}{l}0.01-0.45 \mathrm{mg} / \mathrm{kg} \\
1 \mathrm{~min}\end{array}$ & $\begin{array}{l}\text { Three compart- } \\
\text { ment mammil- } \\
\text { lary model }\end{array}$ & 35.3 & $1.49 \pm 1.9$ & Not reported \\
\hline $\begin{array}{l}\text { Schüttler et al. } \\
\text { (2020) [10] }\end{array}$ & $\begin{array}{l}\text { Healthy } \\
\text { volunteers } \\
(n=20)\end{array}$ & Remimazolam & $\begin{array}{l}5 \mathrm{mg} / \mathrm{min} \text { for } 5 \mathrm{~min} \text {, fol- } \\
\text { lowed by } 3 \mathrm{mg} / \mathrm{min} \text { for } \\
15 \mathrm{~min} \text { and } 1 \mathrm{mg} / \mathrm{min} \\
\text { for } 15 \mathrm{~min}\end{array}$ & $\begin{array}{l}\text { Three compart- } \\
\text { ment mammil- } \\
\text { lary model }\end{array}$ & $35.4 \pm 4.2$ & $1.15 \pm 0.12$ & $70.0 \pm 10.0$ \\
\hline $\begin{array}{l}\text { Sheng et al. } \\
(2020)[11]\end{array}$ & $\begin{array}{l}\text { Healthy } \\
\text { volunteers } \\
(n=62)\end{array}$ & $\begin{array}{l}\text { Remimazolam } \\
\text { Midazolam } \\
\text { Placebo }\end{array}$ & $\begin{array}{l}0.025-0.4 \mathrm{mg} / \mathrm{kg} \\
\text { (SAD study) } \\
0.2 \mathrm{mg} / \mathrm{kg} / \mathrm{min}, \text { followed } \\
\text { by } 1-2 \mathrm{mg} / \mathrm{kg} / \mathrm{h} \\
\text { (infusion study) }\end{array}$ & $\begin{array}{l}\text { Noncompart- } \\
\text { mental model }\end{array}$ & $\begin{array}{l}900.0-1,739.0 \\
\left(^{(S A D ~ s t u d y)}{ }^{\dagger}\right. \\
160.0-229.0 \\
\text { (infusion study) }\end{array}$ & $\begin{array}{c}16.9-20.0 \\
(\mathrm{SAD} \text { study) } \\
0.8-1.3 \\
\text { (infusion study) }\end{array}$ & $34.1-59.8$ \\
\hline
\end{tabular}

Data are presented as the mean $\pm \mathrm{SD}$ or range. SAD: single ascending dose, PK: pharmacokinetics, $\mathrm{V}_{\mathrm{ss}}$ : steady-state volume of distribution, $\mathrm{CL}$ : clearance. ${ }^{*}$ Remimazolam tosylate, a slightly different salt form of remimazolam, was used. ${ }^{\dagger} \mathrm{The}$ unit is $\mathrm{ml} / \mathrm{kg}$. ${ }^{\ddagger} \mathrm{The} u n i t$ is $\mathrm{ml} / \mathrm{min} / \mathrm{kg}$.

no harmful effects on metabolic activity and integrity of liver cells [13].

Remimazolam is predominantly excreted in urine. After $24 \mathrm{~h}$ of remimazolam injection ( 0.2 or $0.3 \mathrm{mg} / \mathrm{kg} \mathrm{IV}$ ), more than $80 \%$ of the dose was detected in urine as a metabolite and less than $1 \%$ of the original dose was detected as unchanged drug [14]. The plasma protein binding of remimazolam was approximately $92 \%$, predominantly serum albu$\min [1]$.

The simulated mean CSHT, which is the time required for the drug plasma concentration to decrease by $50 \%$ after each infusion stop, obtained from pharmacologic analysis has revealed that the offset of remimazolam after the cessation of infusion was faster than that of midazolam but similar to that of propofol $[15,16]$. After $3 \mathrm{~h}$ of constant rate infusion, the CSHT of remimazolam was approximately $7.5 \mathrm{~min}$ vs. 40 min and $7.5 \mathrm{~min}$ for midazolam and propofol, respectively $[15,17]$. Notably, the CSHT of remimazolam appeared to be relatively independent of the infusion duration, reaching its maximum after $2 \mathrm{~h}$ of constant rate infusion, whereas the CSHT of midazolam increased as the duration of infusion increased [17].

One important factor to consider is how the pharmacokinetic properties of remimazolam are altered in various populations, particularly in elderly patients and those with renal or hepatic impairment. The pharmacokinetic properties were not significantly different between the elderly (median age: 66.0 years) vs. young (median age: 21.0 years) patients
[18], patients with normal renal function (estimated glomerular filtration rate $[\mathrm{eGFR}] \geq 90 \mathrm{ml} / \mathrm{min} / 1.73 \mathrm{~m}^{2}$ ) vs. those with end-stage renal failure (eGFR $<15 \mathrm{ml} / \mathrm{min} / 1.73 \mathrm{~m}^{2}$ ), and patients with normal hepatic function vs. those with mild/moderate hepatic dysfunction (Child-Pugh class A and B) [19]. In contrast, the area under the concentration-time curve value from zero to infinity $\left(\mathrm{AUC}_{0 \text {-inf }}\right)$ was higher and clearance was lower in patients with severe hepatic impairment (Child-Pugh class C) than those in normal healthy volunteers (38.1\%, both). Consequently, remimazolam exposure may be increased, and elimination may be prolonged in patients with severe hepatic impairment; therefore, careful dosage adjustment is recommended for these patients [19]. However, these pharmacokinetic analyses were performed using sparse data from small populations; hence, large-scale randomized controlled studies are needed to clarify the safety profile of remimazolam in the aforementioned populations.

\section{Pharmacodynamics}

IV administration of remimazolam enhances the activity of $\gamma$-subunit-containing $\mathrm{GABA}_{\mathrm{A}}$ receptors, and consequently initiates cell membrane hyperpolarization and subsequent neural activity inhibition via an increase in chloride influx [20]. Similar to midazolam, remimazolam enhances $\mathrm{GABA}_{\mathrm{A}}$ currents in cells stably transfected with $\mathrm{GABA}_{\mathrm{A}}$ receptor subtypes ( $\alpha 1, \alpha 2, \alpha 3$, or $\alpha 5$ ); it does not have clear selectivity for 
different subtypes [5].

Pharmacodynamic analyses of sedative effect of remimazolam based on various parameters such as the electroencephalogram (EEG) beta ratio, bispectral index (BIS), Narcotrend index, and Modified Observer's Assessment of Alertness/Sedation (MOAA/S) scores [21] showed a rapid onset and offset and dose-related depth and duration of sedation (Table 2) $[8-11,17,22]$. In a single ascending-dose study of healthy volunteers, fast onset and dose-dependent sedation assessed by MOAA/S scores were observed after IV injection of remimazolam at a concentration of $0.5 \mathrm{mg}$ or higher. In addition, $0.075-0.20 \mathrm{mg} / \mathrm{kg}$ of IV remimazolam induced more rapid peak sedation (within 1-4 min after injection) and deeper sedation than $0.075 \mathrm{mg} / \mathrm{kg}$ of IV midazolam (MOAA/S score < 2 vs. 3-4, respectively) as well as faster median recovery time (5-20 min vs. $40 \mathrm{~min}$, respectively) [8]. Later, a phase I study found that a continuous remimazolam infusion (induction with $0.2 \mathrm{mg} / \mathrm{kg}$ of remimazolam over 1 min and subsequent maintenance with $1.0 \mathrm{mg} / \mathrm{kg} / \mathrm{h}$ for $2 \mathrm{~h}$ ) resulted in deeper sedation and more rapid recovery than those of midazolam $(0.15 \mathrm{mg} / \mathrm{kg}$ over $1 \mathrm{~min}$ and subsequent 2-h maintenance with $0.05 \mathrm{mg} / \mathrm{kg} / \mathrm{h}$ ) [11]. Furthermore, a pharmacodynamic study involving healthy male volunteers found that the MOAA/S score quickly decreased from 5 to < 2 (i.e., full alertness to loss of consciousness) within $5 \mathrm{~min}$ of commencing a 35-min remimazolam infusion, and full alertness was achieved after 19 min of cessation of the infu- sion [10].

A recent time-to-event modeling analysis using various clinical trial data demonstrated that the non-cumulative sedative effect of remimazolam was observed for approximately $9 \mathrm{~h}$ even when administered during general anesthesia [23]. These results could be explained by the rapid offset and short CSHT of remimazolam [10].

\section{Effects on EEG-derived hypnotic index}

In modern anesthesia practice, processed EEG-based hypnotic depth indicators have been widely used to assess the sedative effect of anesthetic drugs, particularly for IV anesthetics because of greater interindividual variability than inhalation anesthetics [24]. Thus, assessment of the effects of remimazolam on EEG-derived hypnotic indices during anesthesia is a crucial practical issue.

A previous study revealed that the EEG changes after IV bolus of midazolam ( 0.2 or $0.3 \mathrm{mg} / \mathrm{kg}$ ) were predominantly beta activation, particularly in the frontal lobe, and these resulted in BIS values remaining around 60 [25]. At present, the EEG changes produced by remimazolam have not been fully clarified. In a phase I study of 20 healthy volunteers, the EEG changes during remimazolam infusion were characterized by an initial transient power increase in the beta frequency band and a subsequent power increase in the delta frequency band; isoelectric EEG patterns or burst suppression were not evident [22].

Table 2. Summary of the Major Pharmacodynamic Parameters of Remimazolam in Healthy Volunteers

\begin{tabular}{|c|c|c|c|c|c|c|c|}
\hline Study (yr) & $\begin{array}{c}\text { Study } \\
\text { population }\end{array}$ & Treatment group & $\begin{array}{c}\text { Remimazolam dose and } \\
\text { duration }\end{array}$ & PD measure & $\begin{array}{l}\text { Time to onset } \\
\text { (min) }\end{array}$ & $\begin{array}{c}\text { Time to peak } \\
\text { sedation (min) }\end{array}$ & $\begin{array}{c}\text { Mean recovery } \\
\text { time (min) }\end{array}$ \\
\hline $\begin{array}{l}\text { Antonik et al. } \\
(2012)[8] \\
\text { Wiltshire et al. } \\
(2012)[17]\end{array}$ & $\begin{array}{l}\text { Healthy } \\
\text { volunteers } \\
(n=72)\end{array}$ & $\begin{array}{l}\text { Remimazolam } \\
\text { Midazolam } \\
\text { Placebo }\end{array}$ & $\begin{array}{l}0.01-0.3 \mathrm{mg} / \mathrm{kg} \\
1 \mathrm{~min}\end{array}$ & MOAA/S, BIS & $\leq 1$ & $1-4$ & $5.5-31.5$ \\
\hline $\begin{array}{l}\text { Zhou et al. } \\
\text { (2018) [9]* }\end{array}$ & $\begin{array}{l}\text { Healthy } \\
\text { volunteers } \\
(n=65)\end{array}$ & $\begin{array}{l}\text { Remimazolam* } \\
\text { Midazolam }\end{array}$ & $\begin{array}{l}0.01-0.45 \mathrm{mg} / \mathrm{kg} \\
1 \mathrm{~min}\end{array}$ & MOAA/S, BIS & $\leq 2$ & $\leq 4$ & 21.5 \\
\hline $\begin{array}{l}\text { Schüttler et al. } \\
(2020)[10] \\
\text { Eisenried et al. } \\
(2020)[22]\end{array}$ & $\begin{array}{l}\text { Healthy } \\
\text { volunteers } \\
(n=20)\end{array}$ & Remimazolam & $\begin{array}{l}5 \mathrm{mg} / \mathrm{min} \text { for } 5 \mathrm{~min} \text {, fol- } \\
\text { lowed by } 3 \mathrm{mg} / \mathrm{min} \text { for } \\
15 \mathrm{~min} \text { and } 1 \mathrm{mg} / \mathrm{min} \\
\text { for } 15 \mathrm{~min}\end{array}$ & $\begin{array}{l}\text { MOAA/S, BIS, EEG } \\
\text { beta ratio, Nar- } \\
\text { cotrend index }\end{array}$ & $\leq 5$ & $5.0 \pm 1.0$ & $19 \pm 7$ \\
\hline $\begin{array}{l}\text { Sheng et al. } \\
(2020)[11]\end{array}$ & $\begin{array}{l}\text { Healthy } \\
\text { volunteers } \\
(n=62)\end{array}$ & $\begin{array}{l}\text { Remimazolam } \\
\text { Midazolam } \\
\text { Placebo }\end{array}$ & $\begin{array}{l}0.025-0.4 \mathrm{mg} / \mathrm{kg} \\
\text { (SAD study) } \\
0.2 \mathrm{mg} / \mathrm{kg} / \mathrm{min} \text {, then } \\
1-2 \mathrm{mg} / \mathrm{kg} / \mathrm{h} \\
\text { (Infusion study) }\end{array}$ & MOAA/S, BIS & $\leq 1$ & $1-2$ & $12.3-25.0$ \\
\hline
\end{tabular}

Data are presented as the mean \pm SD or range. SAD: single ascending dose, PD: pharmacodynamics, MOAA/S: Modified Observer's Assessment of Alertness/Sedation, BIS: bispectral index, EEG: electroencephalogram. *Remimazolam tosylate, a slightly different salt form of remimazolam, was used. 
The BIS algorithm was optimized to yield an approximately linear and monotonic response to increasing doses of propofol. In contrast, the depth of sedation and the BIS index were weakly correlated for midazolam than for propofol [26]. Moreover, the databases used to develop the BIS index did not include EEG data from patients under remimazolam anesthesia. To date, the proper range of the EEG-derived hypnotic index for remimazolam anesthesia have not been clearly demonstrated [2]. Furthermore, the Narcotrend index, one of the EEG-derived hypnotic indices, exhibited a relatively weak relationship with the MOAA/S score in volunteers receiving remimazolam [22].

Consequently, further studies investigating the characteristics of EEG induced by remimazolam and the effects of remimazolam on EEG-derived hypnotic indices are required to determine appropriate levels of hypnosis during anesthesia and sedation with remimazolam.

\section{Target-controlled infusion (TCl)}

Target-controlled infusion is a method of infusing IV drugs while maintaining a user-defined predicted drug concentration. It has been used for more than two decades in clinical anesthesia practice to administer hypnotics and opioids [27]. In particular, TCI is a well-established technique for propofol sedation and anesthesia. The TCI system is also available for administration of remifentanil, an ultra-short-acting drug, even if the benefit of TCI is less obvious compared to that of manual infusion [28]. There is also a controversy about TCI application to remimazolam, another ultra-short-acting drug with a short half-life, high clearance, and $\mathrm{V}_{\mathrm{ss}}$, which would usually be titrated by adjusting the infusion rate [22,29].

However, a previous trial revealed that in simulations of TCI with several anesthetics, steady-state concentration after remimazolam infusion and propofol infusion was achieved after approximately $60 \mathrm{~min}$ and more than $60 \mathrm{~min}$, respectively, whereas it took approximately $10 \mathrm{~min}$ after remifentanil infusion [22]. These findings indicate that the clinical application of TCI for the administration of remimazolam would be reasonable and beneficial [22,29]. In principle, pharmacokinetic/pharmacodynamic (PK/PD) parameters estimated using a multi-compartment mammillary model are required to administer drugs via the TCI method using the currently available equipment [29]. A PK/PD model for remimazolam using a multi-compartment mammary model has been introduced. However, this model has been developed in 20 healthy male volunteers only [10,22]. The PK/PD model only applies when the clinical conditions and patient characteristics match those of the subjects in the model development group. Consequently, the above-mentioned model is also not suitable for administration of remimazolam via the TCI method in various surgical populations $[10,22]$. Even though a population PK/PD model using a multi-compartment mammillary model was constructed in surgical patients, this model is not suitable for the currently available TCI system because the clearance parameter was estimated to change over time [30]. Therefore, new compartmental PK/PD models should be developed in diverse populations and clinical settings for wide applications of TCI to administer remimazolam.

\section{CLINICAL APPLICATIONS OF REMIMAZOLAM}

\section{Remimazolam for procedural sedation}

Procedural sedation is used to allow effective completion of diagnostic or therapeutic procedures that may be uncomfortable for patients or painful [31]. It is thought that ideal characteristics of the hypnotic agents used for procedural sedation include fast onset of action and recovery, minimal residual sedation, ease of use, and few adverse effects [7]. The major characteristics of three widely used sedatives in modern anesthesia practice are summarized in Table 3. BDZ sedatives, including midazolam that is considered to be the gold standard, have been frequently used because of their faster onset and high amnestic potential. However, active metabolite of midazolam is a potent sedative [7] that may prolong the sedation time. In contrast, remimazolam has a rapidly hydrolyzed ester linkage that produces an inactive metabolite; therefore, it seems to be an ideal sedative in terms of minimal residual sedation and organ-independent metabolism.

In comparison to midazolam, remimazolam as a sedative for procedural sedation (typically supplemented by an opioid) produces rapid-onset sedation and clear-headed recovery with low liability for blood pressure perturbation and respiratory depression [7,32-34]. In a phase III study comparing the efficacy and safety of remimazolam with those of midazolam, a total of 461 patients who underwent colonoscopy were randomly assigned to one of the three study arms (5 mg remimazolam with supplementary doses of $2.5 \mathrm{mg}$ remimazolam, midazolam, or placebo plus midazolam res- 
Table 3. Major Characteristics of Propofol, Midazolam, and Remimazolam as Intravenous Hypnotics for Anesthesia and Sedation

\begin{tabular}{lccc}
\hline \multicolumn{1}{c}{ Characteristics } & Propofol & Midazolam & Remimazolam \\
\hline Ready-to-use injectable formulation & + & + & - \\
Free from pain on injection & - & + & + \\
Free from liability for cardiovascular and respiratory depression & - & + & + \\
Active metabolite & - & - & - \\
Availability of a reversal agent & + & $3-5$ & + \\
Short context-sensitive half-time & $<1$ & $20-80$ & $1-2$ \\
Onset (min) & 10 & Hepatic & Hydrolysis by tissue esterase \\
Recovery (min) & Hepatic/extrahepatic & 97 & 92 \\
Metabolism & 98 & & 92 \\
Protein binding (\%) & &
\end{tabular}

+ and - represent the presence and absence of the relevant characteristics, respectively.

cue) [33]. Procedural success was defined as the completion of colonoscopy without a rescue sedative; it was achieved in $91.3,25.2$, and $1.7 \%$ of patients receiving remimazolam, midazolam, and placebo plus midazolam rescue, respectively. In addition, compared with those who received midazolam, fewer patients who received remimazolam experienced hypotension and showed faster recovery. These findings indicate that remimazolam can be administered safely for procedural sedation, and it permits fast recovery of neuropsychiatric function compared with midazolam.

Propofol is another sedative frequently used for procedural sedation. Although it has a rapid onset of action and a very short half-life, it precipitates more adverse events, notably possible hypotension, respiratory depression, bradycardia, and pain upon injection. Therefore, there is a need for safer sedatives while ensuring their efficacy [35]. Clinical studies comparing efficacy and safety of remimazolam vs. propofol for procedural sedation suggested that remimazolam was non-inferior in terms of sedative efficacy and exhibited better safety profile than that of propofol [32,34,36]. Chen et al. [32] demonstrated that the procedure success rate in the remimazolam group was similar to that in the propofol group ( $96.91 \%$ vs. $100 \%$, respectively) in 384 patients who underwent colonoscopy [32]. The safety assessment revealed that the total number of adverse events was lower in the remimazolam group than in the propofol group; in particular, injection site pain, increased bilirubin, decreased respiratory rate, and hypoxia were less frequent in patients receiving remimazolam. Similar findings were reported in another non-inferior study performed on patients who underwent upper gastrointestinal endoscopy [34]. In summary, remimazolam and propofol had similar success rates for sedation, but remimazolam had a more favorable safety profile than that of propofol.

In addition, a patient's physical status affects safety and recovery from procedural sedation; a higher American Society of Anesthesiologists (ASA) physical status classification is associated with a higher risk of adverse periprocedural events [37]. A randomized trial involving patients who underwent high-risk colonoscopy found that the efficacy and safety data of remimazolam for procedural sedation of highrisk ASA patients were comparable to those with low-risk ASA [38]. These results indicate remimazolam to be equally efficient and safe for procedural sedation in patients with low- and high-risk ASA.

Taken together, remimazolam can be used as a safe and effective alternative to other widely-used sedatives, such as midazolam and propofol, for IV sedation in patients undergoing various procedures. Further clinical studies with respect to the quality of patient experience, new formulations (for example, intranasal or inhalation), and post-market cost-benefit analyses are important factors in acquiring the widespread use of remimazolam in procedural sedation.

\section{Dosage and administration}

To induce and maintain procedural sedation in adults, remimazolam dosage should be titrated and individualized to achieve the desired clinical response. IV remimazolam was used at a dose of $5 \mathrm{mg}$ over $1 \mathrm{~min}$ for induction. Supplemental IV doses of remimazolam ( $2.5 \mathrm{mg}$ ) over $15 \mathrm{~s}$ with $\geq 2$ min between doses can be given, if required. The recommended dosage should be reduced in patients with ASA class III/IV as follows: an induction dose of 2.5-5 mg remimazolam and top-up doses of 1.25-2.5 mg on the basis of patient's general condition and at the physician's discretion $[39,40]$. 
The prescribing information for remimazolam specifies cautions related to sedation, such as hypoxia, bradycardia, and hypotension [40]. Accordingly, remimazolam should typically be administered by personnel trained in the administration of procedural sedation. Before administration of remimazolam, drugs, personnel, and equipment for monitoring and resuscitation should be prepared. Continuous monitoring of vital signs must be performed during procedural sedation and during the entire recovery period.

\section{Remimazolam for general anesthesia}

The introduction of remimazolam in clinical practice provides a chance to reappraise $\mathrm{BDZ}$ as a principal anesthetic for general anesthesia. IV remimazolam, which was used as the hypnotic component of total intravenous anesthesia (TIVA), was as effective as propofol and showed superior safety profile for the induction and maintenance of general anesthesia in surgical patients with ASA class I/II [41]. Remimazolam ( 6 or $12 \mathrm{mg} / \mathrm{kg} / \mathrm{h}$ IV infusion, $\mathrm{n}=150$ or 150 , respectively) was administered for induction followed by 1 $\mathrm{mg} / \mathrm{kg} / \mathrm{h}$ of initial maintenance dose. A third group of patients received a standard dose regimen of IV propofol (2.0$2.5 \mathrm{mg} / \mathrm{kg} / \mathrm{h}$, followed by $4-10 \mathrm{mg} / \mathrm{kg} / \mathrm{h})(\mathrm{n}=75)$. All patients were administered $0.25-0.5 \mu \mathrm{g} / \mathrm{kg} / \mathrm{min}$ of remifentanil during the entire study period. The primary efficacy endpoint, defined as no rescue sedative requirement, absence of intraoperative awakening or recall, and no body movement, was accomplished in all three groups of patients, thereby achieving noninferiority. The mean time to loss of consciousness and time to extubation were significantly longer for patients treated with remimazolam than in patients treated with propofol (102.0 s for $6 \mathrm{mg} / \mathrm{kg} / \mathrm{h}$ remimazolam and $88.7 \mathrm{~s}$ for $12 \mathrm{mg} / \mathrm{kg} / \mathrm{h}$ remimazolam vs. $78.7 \mathrm{~s}$ for propofol, and 19.2 and $19.2 \mathrm{~min}$ vs. $13.1 \mathrm{~min}$, respectively) [41]. In contrast, compared with propofol, remimazolam regimens exhibited excellent safety profile. The patients in the remimazolam groups (35.3\% and $34.7 \%$, respectively) experienced fewer hypotensive-specific events than those in the propofol group (60.0\%). Fewer patients in the remimazolam groups needed vasopressors ( $40.0 \%$ and $42.7 \%$, respectively) or treatment for bradycardia ( $6.0 \%$ and $6.7 \%$, respectively) than in the propofol group (64.0\% required vasopressors and $9.3 \%$ required treatment for bradycardia).

Subsequent randomized comparative trials confirmed that remimazolam would be a more useful agent as the hypnotic component of TIVA, particularly when used for clini- cally vulnerable patients. Interestingly, both induction regimens ( 6 and $12 \mathrm{mg} / \mathrm{kg} / \mathrm{h}$ ) were equally efficient and safe in 67 high-risk surgical patients [42]. In addition, an unpublished clinical study comparing remimazolam with propofol suggested that remimazolam had better hemodynamic stability and similar hypnotic efficacy in 90 high-risk patients undergoing major cardiac surgery [43]. The induction dosages were 6 or $12 \mathrm{mg} / \mathrm{kg} / \mathrm{h}$ of IV remimazolam ( $\mathrm{n}=34$ and 28 , respectively), followed by maintenance dose of 1-3 mg/ $\mathrm{kg} / \mathrm{h}$. Another group received an induction dosage of 2-2.5 $\mathrm{mg} / \mathrm{kg}$ IV propofol, and was maintained using inhaled sevoflurane (until the start of extracorporeal circulation) and propofol (during extracorporeal circulation) $(n=28)$. The primary endpoint (no requirement for an additional sedative) was achieved in $98 \%$ of patients administered remimazolam and $96 \%$ of those administered propofol. Onset (time to loss of consciousness) and offset (time to extubation) of action were similar in all study groups. Patients who received remimazolam required significantly less dose of norepinephrine than those who were anesthetized using propofol-sevoflurane.

Taken together, early reported clinical data suggest that remimazolam has excellent efficacy and safety profile as a hypnotic component of balanced anesthesia. Notably, at present, a principal reason for considering remimazolam as a hypnotic underpinning of general anesthesia may be its superior hemodynamic stability. However, to gain widespread use, additional clinical studies investigating postoperative delirium and postoperative nausea and vomiting (PONV) and pharmacological interaction with opioid analgesics are required. These clinical data would further help to clarify remimazolam characteristics as an IV anesthetic.

\section{Dosage and administration}

The licensed prescribing information of remimazolam for use in general anesthesia specifies an initial dose of 6 or 12 $\mathrm{mg} / \mathrm{kg} / \mathrm{h}$ for induction, followed by $1 \mathrm{mg} / \mathrm{kg} / \mathrm{h}(2 \mathrm{mg} / \mathrm{kg} / \mathrm{h}$ of maximal infusion rate) for maintenance. The maintenance infusion rate should be adjusted based on the patient's general condition and sedation level.

To date, there have been no published clinical trials of bolus administration of remimazolam to induce anesthesia; thus, bolus administration is not currently recommended for general anesthesia. Therefore, future trials on bolus remimazolam injection for induction of general anesthesia are warranted to establish more practical administration methods [44]. 


\section{Remimazolam for intensive care unit (ICU) sedation}

Because ICU patients are critically ill with essential organ failure (hepatic or renal), the ideal drug of choice in such a scenario would be a short-acting agent with metabolism independent of the liver or kidney. In this respect, remimazolam could theoretically be a promising drug for long-term sedation in ICU patients due to its favorable properties of organ-independent metabolism, minimal accumulation, and availability of a reversal drug [1].

Currently, there is no published clinical evidence on the potential utility of remimazolam in ICU sedation. Several trials are ongoing to investigate the feasibility of long-term sedation with remimazolam in critically ill patients (NCT0461 1425 and NCT04815265).

\section{Flumazenil reversal}

The hypnotic effect of remimazolam can be reversed using flumazenil, which is an antagonist of the positive allosteric modulator effects of BDZs. However, reversal is not possible for several other hypnotics, including propofol [1]. Reversal of the sedative effect of remimazolam by flumazenil has been well described in several previous studies $[6,41]$. For instance, after successive sedation $(\mathrm{MOAA} / \mathrm{S} \leq 3)$ with 0.25 $\mathrm{mg} / \mathrm{kg}$ of IV remimazolam in healthy volunteers who underwent colonoscopy, the median (range) time until the subjects were fully alert (3 consecutive MOAA/S scores of 5 ) was shorter for patients receiving flumazenil $(0.5 \mathrm{mg})$ than for patients in the placebo group (1.0 [1-5] vs. 10.5 [5-52] min, respectively).

To reverse the hypnotic effect of BDZs, $0.2 \mathrm{mg}$ flumazenil is recommended as an initial dose; it should be slowly injected over a 15-s period to avoid possible adverse events (hypertension, tachycardia, and anxiety). If the desired level of consciousness is not achieved after $60 \mathrm{~s}$ of the initial dose, a second dose of $0.2 \mathrm{mg}$ can be injected and repeated at 60 -s intervals up to a maximum of four additional times and a maximum total dose of $1 \mathrm{mg}$. Subsequently, the dosage should be administered according to the patient's response. If re-sedation occurs, repeated doses may be administered at 20-min intervals. For repeated treatment, no more than 1 mg should be administered at any one time, and no more than $3 \mathrm{mg}$ should be injected during any 1 -h period $[45,46]$.

Clinicians should keep in mind that unlike the relationship between rocuronium and sugammadex, the mecha- nism of action of flumazenil against BDZs is merely based on competitive antagonism and that flumazenil has a short terminal half-life of $40-80 \mathrm{~min}[47,48]$. As the plasma flumazenil concentration decreased, the hypnotic effect of remimazolam could re-emerge. Accordingly, after administration of flumazenil, patients should be monitored for re-sedation, respiratory depression, and other persistent or recurrent hypnotic effects for a sufficient time period. In practice, routine administration of flumazenil to reverse remimazolam-induced sedation is not recommended because of the likelihood of rebound sedation $[49,50]$.

\section{Safety concerns and future directions}

Adverse reactions associated with remimazolam during procedural sedation and anesthesia settings are well documented in various clinical studies, which are in accordance with those observed with other classical BDZs. The most common beings are blood pressure and heart rate changes, nausea, and vomiting [33,41]. Additional adverse events include headache, somnolence, and hypoxia [8]. In addition, results of a cardiac electrophysiology study showed that cardiac repolarization is not prolonged by remimazolam, even if a transient heart rate increase may result from a small increase in the QTc interval [51].

When compared with propofol in both sedation and general anesthesia, remimazolam exhibited better safety profile, including a lower incidence of hypotension, less bradycardia treatment requirement, and no pain on injection $[34,36,41]$. However, propofol was less likely to develop PONV.

Another safety issue related to the administration of hypnotics is drug abuse or potential misuse [52]. One study investigating the abuse potential of remimazolam demonstrated that it has a comparable or lower abuse potential than that of midazolam, which is known to be a drug with a low potential for IV abuse [53].

The solubility of remimazolam decreases at $\mathrm{pH}>4.0$; therefore, the package insert instructions specify that it should not be dissolved in an alkaline solution. Accordingly, Ringer's lactate should not be used as a solvent because the drug does not dissolve completely in solution and forms a precipitate [40]. Sasaki et al. [54] reported the formation of white precipitates related to the combined use of remimazolam and Ringer's solution, and examined the effect of remimazolam concentration on precipitate formation. In this experimental study, both types of Ringer's solution (Ringer's lactate [pH 5.9-6.2] and Ringer's acetate [pH 6.0- 
7.5]) formed precipitates with remimazolam. In addition, the authors suggested that if combined use is unavoidable, lower remimazolam concentrations and higher Ringer's solution infusion rates are recommended to prevent precipitate formation.

Although there are several ongoing trials for the potential use of remimazolam in various clinical settings in pediatric patients (NCT04720963, NCT04851717, and NCT04601350), their safety and effectiveness have not yet been established. Therefore, to date, remimazolam for both general anesthesia and sedation is not available in pediatric population.

At present, limited data are available to characterize remimazolam comprehensively as a hypnotic, even though several volunteer studies and clinical trials suggest that remimazolam is well tolerated and effective for procedural sedation and induction and maintenance of general anesthesia. So yet, to ensure patient safety, the careful introduction of this novel hypnotic agent is needed in clinical practice. Future trials with regard to pharmacological interaction with concomitant anesthetics, new formulations, safety profiles in the special population, characteristics of the EEG changes, bolus administration for the induction of anesthesia, PONV, postoperative cognitive impairment, and post-market cost-benefit analyses would be essential for the comprehension of the complete profile of remimazolam.

\section{CONCLUSION}

Remimazolam is a novel ultra-short-acting hypnotic agent invented out of 'a soft drug' development. Its primary benefits include rapid onset/offset, predictable duration of action, metabolism almost unaffected by organ function, availability of a reversal drug, and maintenance of stable hemodynamics, making it a potential drug for use in various clinical practices. However, further clinical studies are essential to comprehensively evaluate the efficacy and safety profile of this drug before its extended application in various clinical settings.

\section{FUNDING}

None.

\section{CONFLICTS OF INTEREST}

No potential conflict of interest relevant to this article was reported.

\section{DATA AVAILABILITY STATEMENT}

Data sharing not applicable to this article as no datasets were generated or analyzed during the current study.

\section{ORCID}

Kyung Mi Kim, https://orcid.org/0000-0001-6893-1539

\section{REFERENCES}

1. Kilpatrick GJ. Remimazolam: non-clinical and clinical profile of a new sedative/anesthetic agent. Front Pharmacol 2021; 12: 690875.

2. Sigel E, Buhr A. The benzodiazepine binding site of $\mathrm{GABA}_{\mathrm{A}} \mathrm{re}-$ ceptors. Trends Pharmacol Sci 1997; 18: 425-9. Erratum in: Trends Pharmacol Sci 1998; 19: 256.

3. Goudra BG, Singh PM. Remimazolam: The future of its sedative potential. Saudi J Anaesth 2014; 8: 388-91.

4. Egan TD. Is anesthesiology going soft?: trends in fragile pharmacology. Anesthesiology 2009; 111: 229-30.

5. Kilpatrick GJ, McIntyre MS, Cox RF, Stafford JA, Pacofsky GJ, Lovell GG, et al. CNS 7056: a novel ultra-short-acting Benzodiazepine. Anesthesiology 2007; 107: 60-6.

6. Worthington MT, Antonik LJ, Goldwater DR, Lees JP, Wilhelm-Ogunbiyi K, Borkett KM, et al. A phase Ib, dose-finding study of multiple doses of remimazolam (CNS 7056) in volunteers undergoing colonoscopy. Anesth Analg 2013; 117: 1093100.

7. Lee A, Shirley M. Remimazolam: a review in procedural sedation. Drugs 2021; 81: 1193-201.

8. Antonik LJ, Goldwater DR, Kilpatrick GJ, Tilbrook GS, Borkett KM. A placebo- and midazolam-controlled phase I single ascending-dose study evaluating the safety, pharmacokinetics, and pharmacodynamics of remimazolam (CNS 7056): part I. Safety, efficacy, and basic pharmacokinetics. Anesth Analg 2012; 115: 274-83

9. Zhou Y, Hu P, Huang Y, Nuoer S, Song K, Wang H, et al. Population pharmacokinetic/pharmacodynamic model-guided dosing optimization of a novel sedative HR7056 in Chinese healthy subjects. Front Pharmacol 2018; 9: 1316. Erratum in: Front Pharmacol 2019; 10: 251.

10. Schüttler J, Eisenried A, Lerch M, Fechner J, Jeleazcov C, Ihmsen $\mathrm{H}$. Pharmacokinetics and pharmacodynamics of remimazolam (CNS 7056) after continuous infusion in healthy male volunteers: part I. Pharmacokinetics and clinical pharmacodynamics. Anesthesiology 2020; 132: 636-51. 
11. Sheng XY, Liang Y, Yang XY, Li LE, Ye X, Zhao X, et al. Safety, pharmacokinetic and pharmacodynamic properties of single ascending dose and continuous infusion of remimazolam besylate in healthy Chinese volunteers. Eur J Clin Pharmacol 2020; 76: 383-91.

12. Sneyd JR. Remimazolam: new beginnings or just a me-too? Anesth Analg 2012; 115: 217-9.

13. Freyer N, Knöspel F, Damm G, Greuel S, Schneider C, Seehofer D, et al. Metabolism of remimazolam in primary human hepatocytes during continuous long-term infusion in a 3-D bioreactor system. Drug Des Devel Ther 2019; 13: 1033-47.

14. Zhou Y, Hu P, Jiang J. Metabolite characterization of a novel sedative drug, remimazolam in human plasma and urine using ultra high-performance liquid chromatography coupled with synapt high-definition mass spectrometry. J Pharm Biomed Anal 2017; 137: 78-83.

15. Masui K. Remimazolam besilate, a benzodiazepine, has been approved for general anesthesia!! J Anesth 2020; 34: 479-82.

16. Wesolowski AM, Zaccagnino MP, Malapero RJ, Kaye AD, Urman RD. Remimazolam: pharmacologic considerations and clinical role in anesthesiology. Pharmacotherapy 2016; 36: 1021-7.

17. Wiltshire HR, Kilpatrick GJ, Tilbrook GS, Borkett KM. A placebo- and midazolam-controlled phase I single ascending-dose study evaluating the safety, pharmacokinetics, and pharmacodynamics of remimazolam (CNS 7056): part II. Population pharmacokinetic and pharmacodynamic modeling and simulation. Anesth Analg 2012; 115: 284-96.

18. Doi M. Remimazolam. J Jpn Soc Clin Anesth 2014; 34: 860-6.

19. Stöhr T, Colin PJ, Ossig J, Pesic M, Borkett K, Winkle P, et al. Pharmacokinetic properties of remimazolam in subjects with hepatic or renal impairment. Br J Anaesth 2021; 127: 415-23.

20. Rogers WK, McDowell TS. Remimazolam, a short-acting GAB$A_{A}$ receptor agonist for intravenous sedation and/or anesthesia in day-case surgical and non-surgical procedures. IDrugs 2010; 13: 929-37.

21. Chernik DA, Gillings D, Laine H, Hendler J, Silver JM, Davidson $\mathrm{AB}$, et al. Validity and reliability of the Observer's Assessment of Alertness/Sedation Scale: study with intravenous midazolam. J Clin Psychopharmacol 1990; 10: 244-51.

22. Eisenried A, Schüttler J, Lerch M, Ihmsen H, Jeleazcov C. Pharmacokinetics and pharmacodynamics of remimazolam (CNS 7056) after continuous infusion in healthy male volunteers: part II. Pharmacodynamics of electroencephalogram effects. Anesthesiology 2020; 132: 652-66.

23. Lohmer LL, Schippers F, Petersen KU, Stoehr T, Schmith VD. Time-to-event modeling for remimazolam for the indication of induction and maintenance of general anesthesia. J Clin Phar- macol 2020; 60: 505-14.

24. Checketts MR, Alladi R, Ferguson K, Gemmell L, Handy JM, Klein AA, et al. Association of Anaesthetists of Great Britain and Ireland. Recommendations for standards of monitoring during anaesthesia and recovery 2015: Association of Anaesthetists of Great Britain and Ireland. Anaesthesia 2016; 71: 8593.

25. Miyake W, Oda Y, Ikeda Y, Hagihira S, Iwaki H, Asada A. Electroencephalographic response following midazolam-induced general anesthesia: relationship to plasma and effect-site midazolam concentrations. J Anesth 2010; 24: 386-93.

26. Ibrahim AE, Taraday JK, Kharasch ED. Bispectral index monitoring during sedation with sevoflurane, midazolam, and propofol. Anesthesiology 2001; 95: 1151-9.

27. Absalom AR, Glen JI, Zwart GJ, Schnider TW, Struys MM. Target-controlled infusion: a mature technology. Anesth Analg 2016; 122: 70-8.

28. Sneyd JR. Remifentanil manual versus target-controlled infusion. Anesth Analg 2003; 97: 300; author reply 300.

29. Sneyd JR, Rigby-Jones AE. Remimazolam for anaesthesia or sedation. Curr Opin Anaesthesiol 2020; 33: 506-11.

30. Zhou J, Leonowens C, Ivaturi VD, Lohmer LL, Curd L, Ossig J, et al. Population pharmacokinetic/pharmacodynamic modeling for remimazolam in the induction and maintenance of general anesthesia in healthy subjects and in surgical subjects. J Clin Anesth 2020; 66: 109899.

31. Hinkelbein J, Lamperti M, Akeson J, Santos J, Costa J, De Robertis E, et al. European Society of Anaesthesiology and European Board of Anaesthesiology guidelines for procedural sedation and analgesia in adults. Eur J Anaesthesiol 2018; 35: 6-24.

32. Chen S, Wang J, Xu X, Huang Y, Xue S, Wu A, et al. The efficacy and safety of remimazolam tosylate versus propofol in patients undergoing colonoscopy: a multicentered, randomized, positive-controlled, phase III clinical trial. Am J Transl Res 2020; 12: 4594-603.

33. Rex DK, Bhandari R, Desta T, DeMicco MP, Schaeffer C, Etzkorn $\mathrm{K}$, et al. A phase III study evaluating the efficacy and safety of remimazolam (CNS 7056) compared with placebo and midazolam in patients undergoing colonoscopy. Gastrointest Endosc 2018 88: 427-37. e6.

34. Chen SH, Yuan TM, Zhang J, Bai H, Tian M, Pan CX, et al. Remimazolam tosilate in upper gastrointestinal endoscopy: a multicenter, randomized, non-inferiority, phase III trial. J Gastroenterol Hepatol 2021; 36: 474-81.

35. Oka S, Satomi H, Sekino R, Taguchi K, Kajiwara M, Oi Y, Kobayashi R. Sedation outcomes for remimazolam, a new benzodiazepine. J Oral Sci 2021; 63: 209-11. 
36. Zhang X, Li S, Liu J. Efficacy and safety of remimazolam besylate versus propofol during hysteroscopy: single-centre randomized controlled trial. BMC Anesthesiol 2021; 21: 156. Erratum in: BMC Anesthesiol 2021; 21: 173.

37. Enestvedt BK, Eisen GM, Holub J, Lieberman DA. Is the American Society of Anesthesiologists classification useful in risk stratification for endoscopic procedures? Gastrointest Endosc 2013; 77: 464-71.

38. Rex DK, Bhandari R, Lorch DG, Meyers M, Schippers F, Bernstein D. Safety and efficacy of remimazolam in high risk colonoscopy: a randomized trial. Dig Liver Dis 2021; 53: 94-101.

39. PAION, AG. Investigator's brochure remimazolam (CNS 7056). Ver 13.0. Ver 13.0. London, PAION, AG. 2018.

40. Acacia Pharma. BYFAVOTM (remimazolam): US prescribing information [Internet]. 2021 [2021 Nov 10]. Available from: https://acaciapharma.com/news/2020/07/acacia-pharma-announces-us-fda-approval-of-byfavo-remimazolam-for-injection-for-the-induction-and-maintenance-of-procedural-sedation.

41. Doi M, Morita K, Takeda J, Sakamoto A, Yamakage M, Suzuki T. Efficacy and safety of remimazolam versus propofol for general anesthesia: a multicenter, single-blind, randomized, parallel-group, phase IIb/III trial. J Anesth 2020; 34: 543-53.

42. Doi M, Hirata N, Suzuki T, Morisaki H, Morimatsu H, Sakamoto A. Safety and efficacy of remimazolam in induction and maintenance of general anesthesia in high-risk surgical patients (ASA Class III): results of a multicenter, randomized, double-blind, parallel-group comparative trial. J Anesth 2020; 34: 491-501.

43. Probst S, Bevilacqua C, Eibel S, Müller A, Wahlers S, Soehngen M. Difference in vasopressor use and usage patterns in patients undergoing cardiac surgery with remimazolam versus propofol/sevoflurane for general anesthesia. Paper presented at: Anesthesiology 2015 Annual Meeting; 2015 Oct 24-28; San
Diego, CA, USA. Schaumburg (IL): American Society of Anesthesiologists, 2015. p. A4025.

44. Hirata N, Hayamizu K, Yamakage M. How to administer remimazolam for anesthesia induction. J Anesth 2020; 34: 962.

45. Karavokiros KA, Tsipis GB. Flumazenil: a benzodiazepine antagonist. DICP 1990; 24: 976-81.

46. Brogden RN, Goa KL. Flumazenil. A reappraisal of its pharmacological properties and therapeutic efficacy as a benzodiazepine antagonist. Drugs 1991; 42: 1061-89. Erratum in: Drugs 1992; 43: 442.

47. Klotz U, Kanto J. Pharmacokinetics and clinical use of flumazenil (Ro 15-1788). Clin Pharmacokinet 1988; 14: 1-12.

48. Sivilotti ML. Flumazenil, naloxone and the 'coma cocktail'. Br J Clin Pharmacol 2016; 81: 428-36.

49. Yamamoto T, Kurabe M, Kamiya Y. Re-sleeping after reversal of remimazolam by flumazenil. J Anesth 2021; 35: 322.

50. Yamamoto T, Kurabe M, Kamiya Y. A mechanism of re-sedation caused by remimazolam. J Anesth 2021; 35: 467-8.

51. Kleiman RB, Darpo B, Thorn M, Stoehr T, Schippers F. Potential strategy for assessing QT/QTc interval for drugs that produce rapid changes in heart rate: electrocardiographic assessment of the effects of intravenous remimazolam on cardiac repolarization. Br J Clin Pharmacol 2020; 86: 1600-9.

52. Chen W, Chen S, Huang Y. Induction and maintenance of procedural sedation in adults: focus on remimazolam injection. Expert Rev Clin Pharmacol 2021; 14: 411-26.

53. Schippers F, Pesic M, Saunders R, Borkett K, Searle S, Webster L, et al. Randomized crossover trial to compare abuse liability of intravenous remimazolam versus intravenous midazolam and placebo in recreational central nervous system depressant users. J Clin Pharmacol 2020; 60: 1189-97.

54. Sasaki H, Hoshijima H, Mizuta K. Ringer's acetate solution-induced precipitation of remimazolam. Br J Anaesth 2021; 126: e87-9. 